\title{
Optimization Methods and Algorithms for Calculating the Construction of Non-Rigid Pavement for Technological Quarries Roads
}

\author{
Anna Tailakova ${ }^{1, *}$, and Alexander Pimonov ${ }^{1,2}$ \\ ${ }^{1}$ T.F. Gorbachev Kuzbass State Technical University, Kemerovo, 650000, Russian Federation \\ ${ }^{2}$ Institute of Economics and Industrial Engineering, Siberian Branch of the Russian Academy of \\ Sciences, Novosibirsk, 630090, Russian Federation
}

\begin{abstract}
Previously developed by the authors of the optimization model for calculating the construction of non-rigid pavement of public roads is proposed to be used for the calculation of the construction of pavement technological career roads. The article describes the optimization methods and algorithms for calculating the construction of pavement. Possibilities of using methods of coordinate-wise descent, multi-start, dynamic programming for the selection of the optimal construction of pavement are presented. Application of genetic algorithms for the decision of an optimization problem of calculation of a construction of pavements on the basis of comparison of their efficiency with efficiency of search methods is proved. Described results of computational experiment of selection of genetic algorithm operators to reduce the volume of calculations and ensure the stability of the results.
\end{abstract}

\section{Introduction}

Quarry roads located on the territory of mining enterprises are constructed for the movement of specialized vehicles and other means of ensuring the technological process of mining. The cost of construction and maintenance of technological quarries roads make up a significant part of the cost of transporting the coal mass. The efficiency of mining transport is also directly dependent on the quality and durability of the haul road and the main element of the pavement. Road clothes of internal roads of industrial enterprises should be constructed in accordance with the basic provisions of the system of normative documents in construction. Typical solutions can significantly reduce the range of search for the most rational construction of pavement, but they solve this problem only partially, because each material of construction has its own cost, and these costs vary depending on the area of construction of the road, the location of bases, quarries, warehouses, the distance of delivery of materials, etc. Ground conditions are also different even with the same structures. It is impossible to take all this into account in standard solutions. The best solutions to minimize the cost of construction of technological quarries roads can be obtained by calculating the construction of pavement on the basis of the optimization models proposed by the authors. These optimization models make it possible to calculate numerically (to determine the thickness of the layers and the volume of the required materials) the minimum cost of materials of construction of non-rigid pavement. Limitations of models are requirements for strength and frost resistance of the calculated

\footnotetext{
*Corresponding author: taa.vtit@kuzstu.ru
} 
construction in accordance with instructions of normative documents on the construction and calculation of road pavement. Optimal construction includes construction, calculation, technical and economic analysis and selection of the most rational construction solution. The software developed by the authors can significantly reduce the time spent on the construction calculation and consider a greater number of options. The most effective is the automated construction of road pavement using the proposed optimization models, methods and algorithms to obtain optimal solutions to the problem (to minimize the cost of construction) under given soil conditions and strength constraints.

Quarries technological roads are natural and technical objects with a layered structure. The task of calculating the construction of the pavement is reduced to the selection of the thickness of their layers in the options outlined in the construction, or to the choice of materials with appropriate deformation and strength characteristics at a given layer thickness and taking into account the requirements of strength. Thus, it is proposed to use two optimization models to solve the problem of selection of road pavement construction:

1) selection of construction by varying the thickness of the structural layers;

2) selection of construction by varying the materials of the structural layers.

Parameters of the problem are characteristics of structural layers, and limitations are strength requirements in accordance with the normative documentation. In this case, the problem of finding the optimal construction of pavements for technological roads is a problem of multidimensional optimization containing nonlinear constraints, and is reduced to minimizing the cost of construction work in terms of the consumption of materials of structural layers. In this case, the objective function is a multi-extremal function, since it can contain both global and several local extrema.

\section{Application of non-linear programming methods of searching the optimal pavement construction}

Due to nonlinear constraints in proposed optimization models, it is impossible to use analytical methods to solve the problem. By discretizing the intervals, the optimization problem can be reduced to a discrete one and solved by a brute force method. The brute force method ensures that the global extremum is found. A complete search of solutions can be implemented using the method of coordinate descent.

Coordinate descent algorithms is based on the universal approach to algorithmic, numerical optimization: solving an optimization problem by solving a sequence of simpler subproblems. Each iterate is found by fixing most of components of the variable vector at their current values and approximately minimizing the objective function with remaining chosen components. Thus, the calculation of all possible variants of the road pavement construction can be made with successive variations in parameters of each layer of the structure. But this approach raises the problem of inefficiency of brute force, associated with its complexity. During the computational experiment it was found that the time of such a method to solve the problem increases exponentially with respect to the size of the input data. So when calculating the construction of the pavement of 3 layers on the basis of the proposed model, it is necessary to calculate more than 1000 variants of possible constructions (depending on the value of the minimum allowable thickness of the structural layers and step), the construction of 5 layers - more than 100,000 [1-2]. Alternatively, the timeliness of coordinate descent for large-scale problems can be also improved by parallelizing the coordinate updates. The multi-start method involves a parallel run of the coordinate descent method from several points of the set of valid solutions. The best result obtained when running parallel processes is chosen as a solution to the problem. Approximate speedup (ideally) is proportional to the number of processors. [3-4]. 


\section{Application of dynamic programming methods for search the optimal construction of pavement}

The solution of the problem of inefficiency of a full search can be the use by dynamic programming methods, so that it is possible to search the optimal construction that meets the requirements of strength. The dynamic programming approach is solving each subproblem only once, thereby reducing the number of computations. This is especially useful in cases where the number of repetitive subtasks increases exponentially. The method of dynamic programming with profile can be used for the task.

With regard to the problem to be solved, this method involves the allocation of the entire space of feasible solutions to several subsets (in accordance with the number of parameters of the problem), fixing all the variable parameters, except one. Thus, the problem of finding the optimal variant of the construction is divided into subtasks of finding the optimal variant of the construction for each of subsets. Then there is a choice of the best option from received. But in the course of the algorithm in several subsets may have same options. Then the caching method is used to exclude the repeated calculation of construction variants.

Depending on the location of the global minimum point of the objective function, the amount of calculations can be significantly reduced. In the worst case, the operation time of the algorithm will be comparable to the time of the coordinate descent method.

Dynamic programming is a well-proven method used when the problem can be solved recursively, which leads to an algorithm with exponential time complexity. If the subproblems are overlapped, then the dynamic programming approach can reduce unnecessary computation, so each subproblem is solved not more than once, and the time complexity of the algorithm is reduced to polynomial. On the other hand, algorithms based on dynamic programming are usually difficult to parallelize, since the subproblems are interdependent, that means each subproblem requires the results of solving the previous subproblem [5-8].

\section{Application of heuristic methods of finding the optimal construction of pavement}

To reduce the volume of calculations, heuristic methods can be used to obtain an acceptable solution to the problem, using numerical methods focused on finding the best solutions from a variety of alternatives, while avoiding a complete search and comparison of all options. The best known evolutionary algorithm is the genetic algorithm. Mathematically, a genetic algorithm can be defined as a stochastic optimization method for discrete optimization problems.

Genetic algorithms (GA) are adaptive search methods used to solve optimization problems. They use both an analogue of the mechanism of genetic inheritance and an analogue of natural selection. At the same time, the biological terminology in a simplified form and the basic concepts of linear algebra are preserved. Main operators of the genetic algorithm are crossing, mutation, selection of parents and selection (selection of chromosomes in a new population). The type of operator plays an important role in the implementation and efficiency of the GA. There are basic forms of operators whose clean use or modernization leads to a GA suitable for a particular task.

During the computational experiment (table. 1) the following GA parameters were set: in the evolutionary model, each variable corresponds to a gene in the chromosome; to represent chromosomes, the decimal coding is used; the fitness function completely repeats the target function of the proposed mathematical model; the population size is 40 chromosomes; the maximum number of generations is 100 . Different combinations of GA 
operators were used to adjust GA. For each case, the HA was run 50 times for each of the 5 test constructions of non-rigid pavement. An indicator of the stability of the genetic algorithm is the ratio of the number of successful runs to the total number of runs of the algorithm, in addition, the number of generations in which the algorithm was stopped is estimated.

The work of the canonical GA involves the generation of the initial population from a given number of chromosomes, with the help of selection, an intermediate array is formed, from which 2 parents are randomly selected. Next, a single-point crossover is made, and created two descendants mutate (single-point mutation) with a given probability. Mutated descendants take the place of their parents. The process continues until the completion criterion of the algorithm is reached. Computational experiment has shown (tab. 1) that this algorithm demonstrates low stability, slowly converges and is not effective in time due to the large number of calls to the objective function to solve the problem of selecting the optimal construction of road pavement.

Table 1. Results of the computational experiment.

\begin{tabular}{|c|c|c|c|c|c|c|}
\hline & & & & & & \\
\hline $\begin{array}{c}\text { Stability } \\
\text { Average } \\
\text { operating time }\end{array}$ & 8.05 & 15.37 & 13.58 & 2.11 & 16.96 & 3.9 \\
\hline $\begin{array}{c}\text { Average number } \\
\text { construation } \\
\text { options }\end{array}$ & 2784 & 3578.82 & 4000 & 691.2 & 4000 & 997.6 \\
\hline $\begin{array}{c}\text { Average number } \\
\text { of calls to the } \\
\text { target function }\end{array}$ & 955.89 & 1221.83 & 3535.62 & 86.45 & 3535.8 & 47.9 \\
\hline $\begin{array}{c}\text { Average } \\
\text { generation of } \\
\text { the result GA }\end{array}$ & 69.6 & 89.635 & 100 & 17.28 & 100 & 12.47 \\
\hline
\end{tabular}

In the canonical genetic algorithm, the fittest individuals do not always pass on to the next generation. This means that the new population does not always contain the chromosome with the highest fitness function value from the previous population. Elite strategy is used to prevent the loss of such individuals. This individual is guaranteed to be included in the new population. The use of an elitist strategy (table. 1) improves the stability of the algorithm for the task of optimizing the pavement construction.

In the canonical genetic algorithm, the selection of chromosomes in the parent pool is random, replacing the method of random selection by ranked selection (table. 1) increases the stability of the algorithm, making the search for the best solution directional. In this selection, individuals of the population are ranked by the values of their fitness function. This can be represented as a sorted list of individuals, ordered in the direction from the most adapted to the least adapted, in which each individual is assigned a number that determines its place in the list and called the rank. The number of copies of each individual introduced into the parent population is calculated by a priori given function depending on the rank of the individual. 
In addition to the ranked selection, tournament selection is possible, where all individuals of the population are divided into subgroups, followed by the choice of individuals with the best fitness in each of them. The population is divided into subgroups of 2 individuals each. Computational experiment has shown (tab. 1) that tournament selection allows to reduce the amount of calculations, but the stability of the algorithm is reduced.

As a result of the limitations of the problem a large number of chromosomes is unsuitable, i.e. such constructions do not meet the criteria of strength according to the normative documentation. To prevent premature degeneration of the population, a genetic algorithm with partial replacement of the population, otherwise called a genetic algorithm with a fixed state was used. This algorithm is characterized by the fact that some of the best chromosomes in the population goes into the next generation without any changes. The reliability of the algorithm increases, but the amount of calculations increases in comparison with the elite strategy [9-10].

One of the problems of using evolutionary computations to optimize multiextremal functions is to get into a local extremum. To solve this problem, it is possible to use hybrid genetic algorithms [11-13]. The idea of hybrid algorithms is to combine a genetic algorithm with some other classical search method suitable for solving this problem. In each generation, all the generated descendants are optimized by the selected method and then entered into a new population. Thus, it turns out that each individual in the population reaches a local optimum, near which it is located. Further being manufactured for normal GA operations: selection of pairs of parents, crossover and mutation. In practice, hybrid algorithms are very successful. This is due to the fact that the probability of getting one of the individuals in the area of global extrema is usually high. After optimization, such an individual will be the solution to the problem. This approach combines the ability to consider multiple solutions with the accuracy of iterative methods to achieve faster convergence, as well as improving the reliability of the algorithm. Thus, hybrid algorithms show a sufficiently high reliability, allowing quickly to find the optimal or close to the optimal solution of the problem [14].

\section{Conclusion}

The use of optimization models and software that implement optimization methods and algorithms in the calculation of pavement allows specialists to eliminate possible errors that arise in the traditional method of construction. Due to the consideration of a large number of options, it is possible to optimize the construction of pavements according to the specified criteria.

Methods of mathematical modeling are a powerful research tool that allows to conduct computational experiments of any complexity, to search for optimal parameters of the system, to identify patterns of behavior of the object under study. In the course of the computational experiment methods of coordinate descent, multi-start method, dynamic programming method were considered. It is proved the efficiency of application of evolutionary computation in combination with exhaustive search methods to optimize the construction of pavements for technological quarries roads, according to the criterion the total value of the materials.

\section{References}

1. R. Impagliazzo. A personal view of average-case complexity (Gazzone, Rome, 1995) 
2. Michael Shi Hao-Jun, Tu. Shenyinying, Xu. Yangyang, Yin. Wotao, UCLA CAM, 16, 67 (2016)

3. R. Marti, C. Ribeiro Celso, Mauricio G. C. Resende, Multi-start methods for combinatorial optimization, 226:1, 8 (2013)

4. R. Marti, R. Aceves, M.T. Leon, J.M. Moreno-Vega, and A. Duarte. Intelligent Multi-Start Methods, 27, 2 (2018)

5. Moshe Sniedovich, Dynamic Programming Foundations and Principles Second Editio (University of Melbourne Melbourne, Australia, 2011)

6. A. Lew, H. Mauch, Studies in Computational Intelligence, 3, 8 (2007)

7. D. P. Bertsekas, Dynamic Programming \& Optimal Control, (Massachusetts Institute of Technology, Boston, 1995)

8. A. Laaksonen, Competitive Programmer's Handbook (Massachusetts Institute of Technology, Boston, 2018)

9. D. Bednárek, M. Brabec, M. Kruliš, Improving matrix-based dynamic programming on massively parallel accelerators, 64, 175 (2017)

10. K. Oliver, Studies in Computational Intelligence, 67, 9 (2017)

11. E.V. Vasileva, V.S. Doroganov, A.B. Piletskaya, Coke and Chemistry, 60:9, 356 (2017)

12. E.V. Vasileva, V.S. Doroganov, A.B. Piletskaya, Coke and Chemistry, 62:2, 47 (2019)

13. E.V. Vasileva, V.S. Doroganov, A.B. Piletskaya, Coke and Chemistry, 62:2, 40 (2019)

14. S. Ganjefar, M. Tofighi, Engineering Applications of Artificial Intelligence, 65, 346 (2017) 